\title{
Quality improvement of manufacturing rolling mill rolls
}

\author{
S. M. Bratan, Dr. Eng., Prof., Head of Dept. "Machine-building technology"l \\ S. I. Roshchupkin, Cand. Eng., Associate Prof., Dept. "Machine-building technology", \\ e-mail: siroshchupkin@sevsu.ru, st.roshchupkin@yandex.ru \\ A. O. Kharchenko, Cand. Eng., Prof., Dept. "Machine-building technology"l \\ S. V. Belousov, Cand. Eng. ${ }^{2}$
}

\section{${ }^{1}$ Sevastopol State University, Sevastopol, Russia \\ ${ }^{2}$ Kuban State Agrarian University named after I. T. Trubilin, Krasnodar, Russia}

\begin{abstract}
At present time grinding operations for rolling mills rolls, providing the final quality parameters, are the most labour-intensive ones. It is caused first of all via their complex stochastic nature, which leads to a spread of quality indicators, lowering of reliability, productivity and efficiency of technological process. Development of the dynamic process models which can adequately describe the changes occurring in the technological system is considered as one of the ways for solving these problems. The models allowing to assess the influence of random components of the tool profile deviations as factors disturbing the grinding process dynamics are presented in the article. This assessment makes it possible to select the dynamic system parameters (such as mass, damping and stiffness) in order to ensure the maximal possible tool life, the permissible spread of the product quality parameters, as well as the maximal efficiency and productivity of the operation.
\end{abstract}

Key words: grinding wheel, rolling mill rolls, grinding process dynamics, mathematical model, roughness, profile deviation, tool life.

DOI: $10.17580 /$ cisisr.2021.02.05

\section{Introduction}

Production facilities in sheet rolling shops at metallurgical works are characterized by strict requirements to rolling mills rolls not only in their processing accuracy, but also in surface cleanness. The quality parameters are finally forming during grinding operations, which are the most responsible in the technological process. The part of cost expenditures for these operations makes $19-27 \%$ in average from the total cost of manufacturing components [1].

Grinding processes have complex stochastic nature [2], which leads to a spread of product quality indicators, reduced reliability, productivity and efficiency of the process.

Band surface roughness decreases with improvement of surface cleanness of rolling rolls in cold rolling. So, tinplate quality improves and tin consumption decreased by $4.2 \%$ during tin-ning when surface roughness of rolling rolls in tinplate production lowers from $R a=1.6-1.25 \mu \mathrm{m}$ to $R a=0.1-0.05 \mu \mathrm{m}$. Such roughness decrease of rolls surface leads to rise of their resistance by $20 \%$ [2].

The rolls of cold rolling mills are usually subjected to grinding in special circular grinding machines which can't provide always required accuracy and surface roughness. Dispersion of quality parameters is caused by wear of abrasive tools and leads to lowering of resource of the components [3]. Multiple previous researches were devoted to development of dynamic models for various abrasive processing processes [4-16], however, they don't take into account the stochastic nature of this process.

The aim of this work is quality improvement of grinded surfaces of cold rolling rolls due to taking into account fluctuation effects on the process of circular external grinding. Corresponding mathematical models are required for this purpose.

(c) Bratan S. M., Roshchupkin S. I., Kharchenko A. O., Belousov S. V., 2021

\section{Mathematical simulation}

The process of circular external grinding can be presented as the equivalent scheme of the dynamic system of interaction between grinding wheel and billet (Fig. 1).

The following system of equations was obtained based on the D'Alembert principle:

$\left\{\begin{array}{c}m_{1} \ddot{x}_{1}+h_{1} \dot{x}_{1}+c_{1} x_{1}+h_{3} \dot{t}_{\mathrm{f}}+c_{3} t_{\mathrm{f}}-h_{1} \dot{S}-c_{1} S=0 \\ m_{2} \ddot{x}_{2}+h_{2} \dot{x}_{2}+c_{2} x_{2}-h_{3} \dot{t}_{f}-c_{3} t_{f}=0,\end{array}\right.$

where $m_{1}, m_{2}-$ deduced masses of billet and grinding wheel respectively; $h_{\mathrm{i}}$ - damping coefficient; $c_{\mathrm{i}}-$ stiffness; $x_{1}$ and $x_{2}$ - coordinates of tool and billet centers respectively; $S$ - distance passed by tool during time $t ; t_{f}$ - actual cutting depth which is determined by the relationship (see Fig. 1):

$t_{f}=R+r-L$,

where $R=R_{0}+\Delta R$ - grinding wheel radius; $R_{0}$ - its initial value; $\Delta R$ - wear value taking into account deviations of tool shape; $r=r_{0}+\Delta r-$ current billet radius; $r_{0}-$ billet radius before grinding; $\Delta r$-material removal; $L=L_{0}+\Delta L=L_{0}+$ $+x_{2}-x_{1}-$ center-to-center distance.

Taking into account the obtained determination of $t_{f}$ and its components, the system of equations (1) is expressed as:

$\left\{\begin{array}{l}m_{1} \ddot{x}_{1}+h_{1}\left(\dot{x}_{1}-\dot{S}\right)+h_{3}\left(\dot{R}+\dot{r}-\dot{x}_{2}+\dot{x}_{1}\right)+c_{1}\left(x_{1}-S\right)+ \\ +c_{3}\left(R_{0}+\Delta R+r_{0}+\Delta r-L_{0}-x_{2}+x_{1}\right)=0 \\ m_{2} \ddot{x}_{2}+h_{2} \dot{x}_{2}+c_{2} x_{2}-h_{3}\left(\dot{R}+\dot{r}-\dot{x}_{2}+\dot{x}_{1}\right)- \\ -c_{3}\left(R_{0}+\Delta R+r_{0}+\Delta r-L_{0}-x_{2}+x_{1}\right)=0 .\end{array}\right.$ 
Let's indicate $y_{1}=x_{1}, y_{2}=y_{1}=x_{1}, y_{2}=\dot{y}_{1}=\dot{x}_{1}, y_{3}=x_{2}$, $y_{4}=\dot{y}_{3}=\dot{x}_{2}$ and present the system of equations (2) in Cauchy normal form [7]:

$$
\left\{\begin{array}{l}
\dot{y}_{1}=y_{2}, \\
\dot{y}_{2}=-\frac{1}{m_{1}}\left[\left(c_{1}+c_{3}\right) y_{1}+\left(h_{1}+h_{3}\right) y_{2}-c_{3} y_{3}-h_{3} y_{4}\right]- \\
-\frac{1}{m_{1}}\left[c_{3}(\Delta R+\Delta r) y_{1}+h_{3}(\dot{R}+\dot{r})\right]+\frac{1}{m_{1}}\left[h_{1} S+c_{1} S\right], \\
\dot{y}_{3}=y_{4}, \\
\dot{y}_{4}=-\frac{1}{m_{2}}\left[\left(c_{2}+c_{3}\right) y_{3}+\left(h_{2}+h_{3}\right) y_{4}-c_{3} y_{1}-h_{3} y_{2}\right]+ \\
+\frac{1}{m_{2}}\left[c_{3}(\Delta R+\Delta r) y_{1}+h_{3}(\dot{R}+\dot{r})\right],
\end{array}\right.
$$

The obtained dynamic description differs from other known descriptions which are characterized by presence of shape variations of wheel and billet, having almost periodical type and generating internal inducing forces.

To describe deviations of a grinding wheel shape, let's use factorization of expression describing wheel radius-vector into Fourier series, taking into account its deviations from the average value (by the angle $\gamma$ ) $R_{0}(x, \tau)=M\{R(\gamma, x, \tau)\}_{\gamma}$ :

$$
\begin{aligned}
& \left.R(\gamma, x, \tau)\right|_{\tau=0}=\left.R_{0}(x, \tau)\right|_{\tau=0}+\sum_{i=1}^{p}\left[A_{R i}(x, \tau) \cdot \cos (\gamma \cdot i+\right. \\
& \left.\left.+\Psi_{i}(x, \tau)\right)\right]\left.\right|_{\tau=0}=\left.R_{0}(x, \tau)\right|_{\tau=0}+\sum_{i=1}^{p}\left[U_{R i}(x, \tau) \cdot \sin (\gamma \cdot i)+\right. \\
& \left.+U_{R i}(x, \tau) \cdot \cos (\gamma \cdot i)\right]\left.\right|_{\tau=0} .
\end{aligned}
$$

Components of sums in the equation (4) are considered as harmonics of spectral presentation of deviation, which is characterized by the complex of amplitudes $A_{R i}(x, \tau)$ and phases $\Psi_{i}(x, \tau)$ or pair of amplitudes for sine $\left(U_{R i}(x, \tau)\right)$ and cosine $\left(W_{R i}(x, \tau)\right)$ for the components of each harmonic.

The following single-valued (within the range of angle variation $0 \leq \gamma<2 \pi$ ) relationships between parameters of these presentations for each fixed value $i$ can be expressed as:
$A_{R i}^{2}(x, \tau)=U_{R i}^{2}(x, \tau)+W_{R i}^{2}(x, \tau)$, and

$\Psi_{R i}(x, \tau)=\arctan \left(\frac{U_{R i}(x, \tau)}{W_{R i}(x, \tau)}\right)$.

Initial vector $\left.R(\gamma, x, \tau)\right|_{\tau=0}$ and wheel wear value $\Delta R(\gamma, x, \tau)$ include regular and random components of deviations from their average values. A radius of average periphery at distance $x$ from a basic plane $R_{0}(x, \tau)$ at the initial moment $\tau=0$ is a random initial parameter of amplitude, and phases of $p$ harmonics at the moment $\tau$ for each $x$ characterize non-random realization of a random function of a grinding wheel surface.

Taking into account the above-mentioned conclusions, we can present the expression (4) in the following form:

$$
\begin{aligned}
& \left.R(\gamma, x, \tau)\right|_{\tau=0}=\left.R_{0}(x, \tau)\right|_{\tau=0}+\sum_{i=1,}^{p}\left[A_{R i} \cdot \cos (\gamma \cdot i+\right. \\
& \left.\left.+\Psi_{i}(x, \tau)\right)\right]\left.\right|_{\tau=0}+\left.\left[A_{R j} \cdot \cos \left(\gamma \cdot \mathrm{j}+\Psi_{\mathrm{j}}(x, \tau)\right)\right]\right|_{\tau=0}
\end{aligned}
$$

or

$$
\begin{aligned}
& \left.R(\gamma, x, \tau)\right|_{\tau=0}=\left.R_{0}(x, \tau)\right|_{\tau=0}+\sum_{i=1,}^{p}\left[U_{R i} \cdot \sin (\gamma \cdot i)+\right. \\
& \left.+U_{R i} \cdot \cos (\gamma \cdot i)\right]\left.\right|_{\tau=0}+\left.A_{R j} \cdot \cos \left(\gamma \cdot j+\Psi_{j}(x, \tau)\right)\right|_{\tau=0},
\end{aligned}
$$

Where the current angle $\gamma=\gamma_{0}+\int_{0}^{t} \omega_{k}(\tau) d \tau$ is determined by the expression $\left(\gamma_{0}-\right.$ initial angle, $\omega_{k}(\tau)-$ angular speed of tool rotation).

As soon as tool operation time increases, its radius decreases due to wear:

$$
R(\gamma, x, \tau)=\left.R(\gamma, x, \tau)\right|_{\tau=0}-\Delta R(\gamma, x, \tau) .
$$

Wear value for tool working surface depends on intensity of its surface wear $S_{R}(\gamma, x, \tau)$ :

$$
\Delta R(\gamma, x, t)=\int_{0}^{t} S_{R}(\gamma, x, \tau) d \tau .
$$

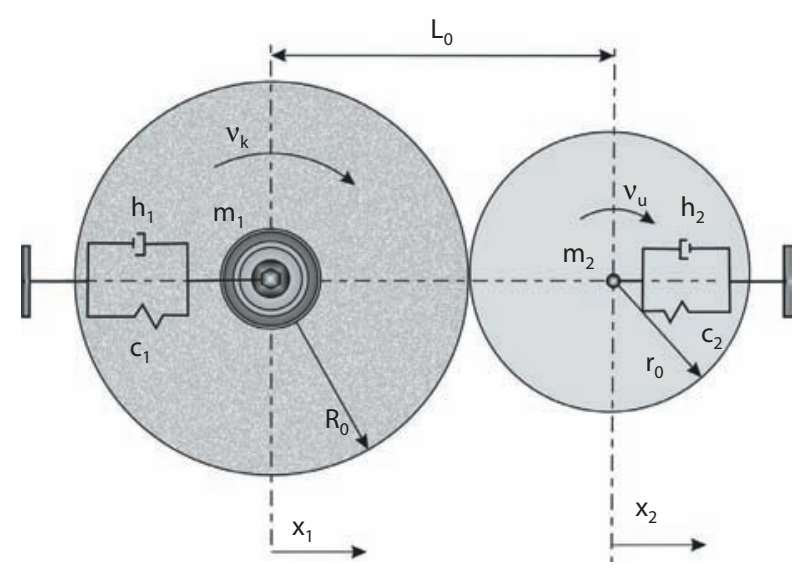

a)

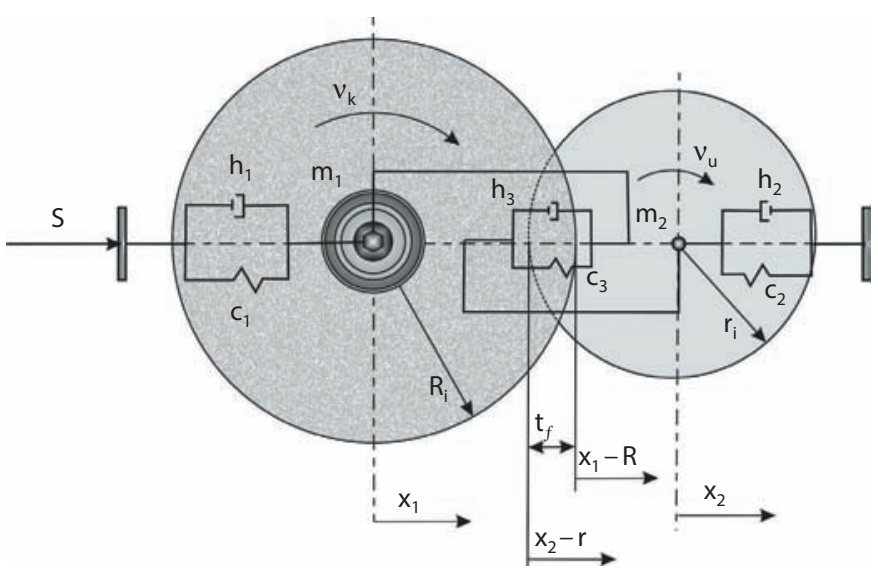

b)

Fig. 1. Dynamic scheme of interaction between grinding wheel and billet: $a-$ at the initial time; $b-$ during grinding 
Mathematical expectation of a random value of radial wear was calculated on the base of properties of linear operators:

$$
\begin{aligned}
& M\{\Delta R(\gamma, x, \tau)\}=M\left\{\int_{0}^{t} S_{R}(\gamma, x, \tau) \cdot d \tau\right\}= \\
& =\int_{0}^{t} M\left\{S_{R}(\gamma, x, \tau)\right\} \cdot d \tau .
\end{aligned}
$$

Let's present wear rate as a functional of $\gamma$, conform to (4), (5):

$$
\begin{aligned}
& S_{R}(\gamma, x, \tau)=\bar{S}(x, \tau)+\sum_{i=1}^{p}\left[U_{S i} \cdot \cos (\gamma \cdot i)+W_{S i} \times\right. \\
& \times \cos (\gamma \cdot i)]+A_{S i}(x, \tau) \cdot \cos \left[\gamma \cdot i+\Psi_{\mathrm{i}}(x, \tau)\right] .
\end{aligned}
$$

Taking into account the expression (9), as a result of integration we obtained a random function of tool radius wear (4):

$\Delta R(\gamma, x, t)=\int_{0}^{t} \bar{S}(x, \tau) \cdot d \tau+\int_{0}^{t} A_{S i}(x, \tau) \cdot \cos \left[\gamma \cdot i+\Psi_{i}(x, \tau)\right] d \tau+$ $+\sum_{i=1}^{p}\left[\int_{0}^{t} U_{S i} \cdot \sin (\gamma \cdot i) \cdot d \tau+\int_{0}^{t} W_{S_{i}} \cdot \cos (\gamma \cdot i) \cdot d \tau\right]$

Integrals of random values $U_{S i}$ and $W_{S i}$ will be the new random values $U_{\Delta R i}$ and $W_{\Delta R i}$, which are also justified by the relationships like (9).

Substituting the obtained functions of the initial radiusvector and wear values in the equation (8), we obtain the relationship for calculation of the current value of tool radius-vector:

$$
\begin{aligned}
& R(\gamma, x, \tau)=R_{0}(x)-\int_{0}^{t} \bar{S}(x, \tau) d \tau+A_{R j}(x) \cdot \cos [\gamma \cdot j+ \\
& \left.+\Psi_{\mathrm{j}}(x)\right]-\int_{0}^{t} A_{S i}(x, \tau) \cdot \cos \left[\gamma \cdot i+\Psi_{i}(x)\right] d \tau+ \\
& +\sum_{i=1}^{p}\left[\left(U_{S i}-U_{\Delta R i}\right) \cdot \sin (\gamma \cdot i)+\left(W_{S i}-W_{\Delta R i}\right) \cdot \cos (\gamma \cdot i)\right]
\end{aligned}
$$

The random components of $i$ th and $j$ th harmonics are remained here under the sum sign, their analysis is conducted similarly to analysis of other random deviations.

The equation (13) includes mathematical expectation of a random function distributed according to the Gauss' law:

$$
M\left\{R_{0}(x)\right\}=\left.m_{R}(x, \tau)\right|_{\tau=0} .
$$

Mathematical expectation of the second component is determined by the expression (10), mathematical expectation of the third and fourth components during whole num- ber of tool rotation periods are equal to zero, and mathematical expectation of the fifth component for the same conditions, for the cases of uniform tool wear is also equal to zero. Based on this we can write down:

$$
M\{R(\gamma, x, t)\}=\left.m_{R}(x, \tau)\right|_{\tau=0}-\int_{0}^{t} m_{S}(x, \tau) d \tau .
$$

Let's divide the right part of the equation (13) to several independent complexes. The first complex (first and second components) determines the average radius-vector at the moment $t$. As soon as variation of radius-vector of a grinding wheel during its resistance period is small, it can't influence on forming of random deviations of its shape. In this connection, the dispersion value for the first complex is determined as:

$$
\begin{aligned}
& \left.K_{1, R}\left(\gamma_{1}-\gamma_{2}\right)\right|_{\gamma_{1}=\gamma_{2}}=M\left\{\left[R_{0}(x)-m_{r}(x)\right] \times\right. \\
& \left.\times\left[\bar{S}(x, t)-m_{S}(x, t)\right]\right\} \cong \sigma_{R_{0}(x)}^{2}+D \cdot\left[\Delta R_{0}(x, \tau)\right] .
\end{aligned}
$$

Correlation functions of the second complex (third and fourth components) are calculated as mathematical expectation of centered values from arguments of $\gamma_{1}$ and $\gamma_{2}$ :

$$
\begin{aligned}
& K_{2, R}\left(\gamma_{1}-\gamma_{2}\right)=M\left\{\left[\left(A _ { j } ( x ) \cdot \operatorname { c o s } \left(j \cdot \gamma_{1}+\right.\right.\right.\right. \\
& \left.+\Psi_{\mathrm{j}}(x)\right)-\int_{0}^{t} A_{S, i}(x, \tau) \cdot \cos \left(i \cdot \gamma_{1}+\right. \\
& \left.\left.+\Psi_{\mathrm{i}}(x, \tau)\right) d \tau\right] \times\left[A _ { j } ( x ) \cdot \operatorname { c o s } \left(j \cdot \gamma_{2}+\right.\right. \\
& \left.\left.\left.+\Psi_{\mathrm{j}}(x)\right)-\int_{0}^{t} A_{S, i}(x, \tau) \cdot \cos \left(i \cdot \gamma_{2}+\Psi_{i}(x, \tau)\right) d \tau\right]\right\}= \\
& =M\left\{\left(A_{j}^{2}(x) \cdot \cos \left(j \cdot \gamma_{1}+\Psi_{j}(x)\right) \cdot \cos \left(j \cdot \gamma_{2}+\Psi_{j}(x)\right)-\right.\right. \\
& -A_{j}(x) \cdot \cos \left(j \cdot \gamma_{1}+\Psi_{j}(x)\right) \cdot \int_{0}^{t} A_{S, i}(x, \tau) \cdot \cos \left(i \cdot \gamma_{2}+\right. \\
& \left.+\Psi_{i}(x, \tau)\right) d \tau-\int_{0}^{\mathrm{t}} A_{S, i}(x, \tau) \cdot \cos \left(i \cdot \gamma_{1}+\Psi_{i}(x, \tau)\right) d \tau \times \\
& \times A_{j}(x) \cdot \cos \left(j \cdot \gamma_{2}+\Psi_{j}(x, \tau)\right) \int_{0}^{t} A_{S, i}(x, \tau) \cdot \cos \left(i \cdot \gamma_{1}+\right. \\
& \left.\left.+\Psi_{i}(x, \tau)\right) d \tau \cdot \int_{0}^{t} A_{S, i}(x, \tau) \cdot \cos \left(i \cdot \gamma_{2}+\Psi_{i}(x, \tau)\right) d \tau\right\}
\end{aligned}
$$

Phase angles $\Psi_{j}$ and $\Psi_{i}$ are uniformly distributed within the interval from 0 to $2 \cdot \pi$ for the established process:

$$
f\left(\Psi_{i}\right)=\left\{\begin{array}{cc}
0 & \forall \Psi_{i}<0 \\
\frac{1}{2 \pi} & \forall 0 \leq \Psi_{i}<2 \pi \quad \forall i=1, \ldots, p \\
0 & \forall \Psi_{i}>2 \pi
\end{array}\right.
$$

The first component from the equation (16) for the conditions (17) is expressed as: 


$$
\begin{aligned}
& K_{2, R}\left(\gamma_{1}-\gamma_{2}\right)=M\left[A_{j}^{2}(x) \cdot \cos \left(j \cdot \gamma_{1}+\Psi_{j}(x, \tau)\right) \times\right. \\
& \left.\times \cos \left(j \cdot \gamma_{2}+\Psi_{j}(x, \tau)\right)\right]=\frac{A_{j}^{2}(x)}{2} \int_{0}^{2 \pi} \cos \left(j \cdot \gamma_{1}+\right. \\
& \left.+\Psi_{j}(x, \tau)\right) \cdot \cos \left(j \cdot \gamma_{2}+\Psi_{j}(x, \tau)\right) f\left(\Psi_{j}\right) d \Psi_{j}= \\
& =\frac{A_{j}^{2}(x)}{2} \cos \left(j \cdot\left(\gamma_{2}-\gamma_{1}\right)\right) .
\end{aligned}
$$

The last component from the equation (16) is determined in the same way for $\Psi_{i}$, which is not depended on $\tau$ :

$$
\begin{aligned}
& M\left\{\int_{0}^{t} A_{S, i}(x, \tau) \cos \left(i \cdot \gamma_{1}+\psi_{i}\right) d \tau \times \int_{0}^{t} A_{S, i}(x, \tau) \times\right. \\
& \left.\times \cos \left(i \cdot \gamma_{2}+\psi_{i}\right) d \tau\right\}=\frac{\cos \left(i \cdot\left(\gamma_{2}-\gamma_{1}\right)\right)}{2} \times \\
& \times\left[\int_{0}^{t} A_{S, i}(x, \tau) \cdot d \tau\right]^{2}
\end{aligned}
$$

Mathematical expectations of the second and third components of the equation (16) are determined via known correlation functions, taking into account the relation between harmonic deviations in tool shape and its wear.

Let's consider several examples which are most often met in the practice. If the initial phase of wear harmonics $\Psi_{i}$ does not depend on the initial phase $\Psi_{j}$, then mathematical expectations of the second and third components are equal to zero. Consequently

$$
\begin{aligned}
& K_{2, R}\left(\gamma_{1}-\gamma_{2}\right)=\frac{A_{j}^{2}(x)}{2} \cos \left(j \cdot\left(\gamma_{2}-\gamma_{1}\right)\right)+ \\
& +\frac{\cos \left(i \cdot\left(\gamma_{2}-\gamma_{1}\right)\right)}{2}\left[\int_{0}^{t} A_{S, i}(x, \tau) d \tau\right]^{2}
\end{aligned}
$$

For the case of the same frequencies which differ only by their phase, i. e. when $i=j$ and $\Psi_{i}=\Psi_{j}+\alpha_{\Psi}$, we shall obtain:

$$
\begin{aligned}
& M\left\{A_{j}(x) \cdot \cos \left(j \cdot \gamma_{1}+\psi_{j}\right) \cdot \cos \left(j \cdot \gamma_{2}+\Psi_{j}+\alpha_{\psi}\right) \times\right. \\
& \times \int_{0}^{t} A_{S i}(x, \tau) d \tau+A_{j}(x) \cdot \cos \left(j \cdot \gamma_{2}+\Psi_{j}\right) \cdot \cos \left(j \cdot \gamma_{1}+\right. \\
& \left.\left.+\Psi_{j}+\alpha_{\psi}\right) \cdot \int_{0}^{t} A_{S i}(x, \tau) d \tau\right\}\left.\right|_{\gamma}=\frac{A_{j}(x)}{4} \cdot \int_{0}^{t} A_{S i}(x, \tau) d \tau \times \\
& \times \int_{0}^{2 \pi}\left[\cos \left(j \cdot\left(\gamma_{1}-\gamma_{2}\right)-\alpha_{\psi}\right)+\cos \left(\left(\gamma_{1}+\gamma_{2}\right) \times\right.\right. \\
& \left.\times j+2 \Psi_{j}+\alpha_{\psi}\right)+\cos \left(\mathrm{j} \cdot \gamma_{2}-j \cdot \gamma_{1}-\alpha_{\psi}\right)+\cos \left(j \cdot \gamma_{2}+\right. \\
& \left.\left.+j \cdot \gamma_{1}+2 \Psi_{j}+\alpha_{\psi}\right)\right] \cdot f\left(\Psi_{i}\right) d \Psi_{j}= \\
& =A_{j}(x) \cdot \cos \left[j \cdot\left(\gamma_{2}-\gamma_{1}\right)\right] \cdot \cos \left(\alpha_{\psi}\right) \cdot \int_{0}^{t} A_{S i}(x, \tau) d \tau .
\end{aligned}
$$

The last component of the equation (13) determines the components of the third complex (if the condition (17) is executed for each harmonics):

$$
\begin{aligned}
& M\left\{A_{k} \cdot \cos \left[\gamma_{1} \cdot k+\Psi_{k}\right] \cdot A_{k} \cdot \cos \left[\gamma_{2} \cdot k+\Psi_{k}\right]\right\}= \\
& =\frac{A_{0 k}^{2}+D\left\{A_{k}\right\}}{2} \cos \left(k \cdot\left(\gamma_{2}-\gamma_{1}\right)\right)
\end{aligned}
$$

The energetic spectrum, which is presented by the components of the equation (21), is determined under the conditions of the equation (17) and distribution of amplitudes $A_{k}$ according to Relay law as follows:

$$
K_{3, R}\left(\gamma_{2}-\gamma_{1}\right)=\sum_{k=1}^{p} \frac{A_{0 k}^{2}+D\left\{A_{k}\right\}}{2} \cdot \cos \left(k \cdot\left(\gamma_{2}-\gamma_{1}\right)\right)
$$

As soon as the examined complexes are independent, their correlation function is calculated as:

$$
\begin{aligned}
& K_{R}\left(\gamma_{2}-\gamma_{1}\right)=\sigma_{p}^{2}(x)+D\left\{\Delta R_{0}(x, \tau)\right\}+\left\{\frac{1}{2} A_{j}^{2}(x)+\right. \\
& \left.+\frac{1}{2}\left[\int_{0}^{t} A_{S j}(x, \tau) d \tau\right]^{2}-A_{j}(x) \cdot \cos \left(\alpha_{\psi}\right) \cdot \int_{0}^{t} A_{S j}(x, \tau) d \tau\right\} \times \\
& \times \cos \left[j \cdot\left(\gamma_{2}-\gamma_{1}\right)\right]+\sum_{k=1}^{p} \frac{A_{0 k}^{2}+D\left\{A_{k}\right\}}{2} \cdot \cos \left(k\left(\gamma_{2}-\gamma_{1}\right)\right) .
\end{aligned}
$$

The obtained complexes for the above-mentioned conditions allow to determine spectral density for each component.

If we accept $\gamma_{1}=\gamma_{2}$ in the equation (23), dispersion of a random value of working surface radius-vector can be determined in the following way:

$$
\begin{aligned}
& K_{R}\left(\gamma_{2}-\gamma_{1}\right)=\sigma_{p}^{2}(x)+D\left\{\Delta R_{0}(x, \tau)\right\}+\frac{1}{2} A_{j}^{2}(x)+ \\
& +\frac{1}{2}\left[\int_{0}^{t} A_{S j}(x, \tau) d \tau\right]^{2}-A_{j}(x) \cdot \cos \left(\alpha_{\psi}\right) \cdot \int_{0}^{t} A_{S j}(x, \tau) d \tau+ \\
& +\sum_{k=1}^{p} \frac{A_{0 k}^{2}+D\left\{A_{k}\right\}}{2} .
\end{aligned}
$$

The following relationship is suggested in the work [3] during analysis of random components of the equation (23) for the $k$ th harmonic:

$K_{3, R}\left(\gamma_{2}-\gamma_{1}\right)=D_{0} \cdot \exp \left(-\alpha_{k} \cdot \omega_{k} \cdot \tau\right) \cdot \cos \left(k \cdot\left(\gamma_{2}-\gamma_{1}\right)\right)$,

where $D_{0}-$ dispersion of heights of shape irregularities for a wheel of $k$ th harmonic; $\alpha_{k}-$ empiric coefficient, taking into account dispersion variation during the period of tool resistance. For the process of flat grinding the $\alpha$ value is usually less than zero.

Based on the functions (23-25), their spectral images, which are determined by Fourier transformation, can be built. 


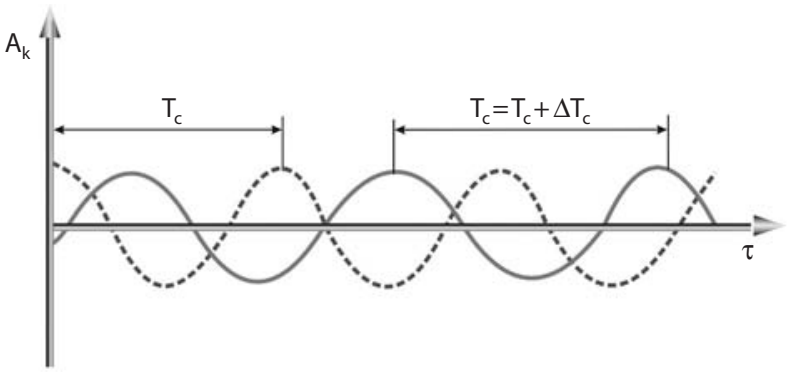

a)

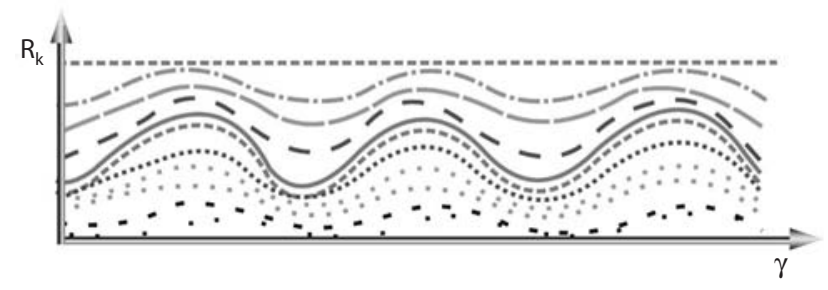

b)

Fig. 2. The scheme of wave forming on billet surface during grinding, taking into account wheel wear during its operation period: $a$ - oscillations of the system "Billet - centers"; $b$ - tool wear scheme

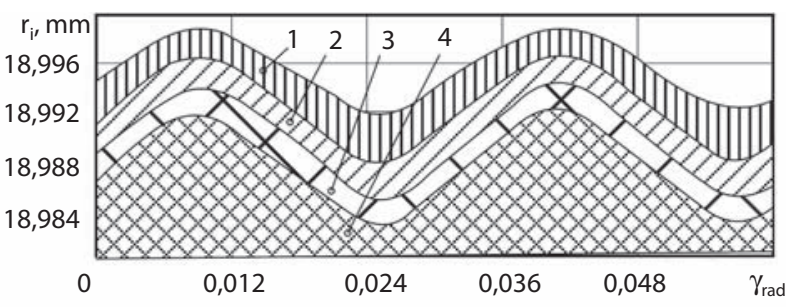

a)

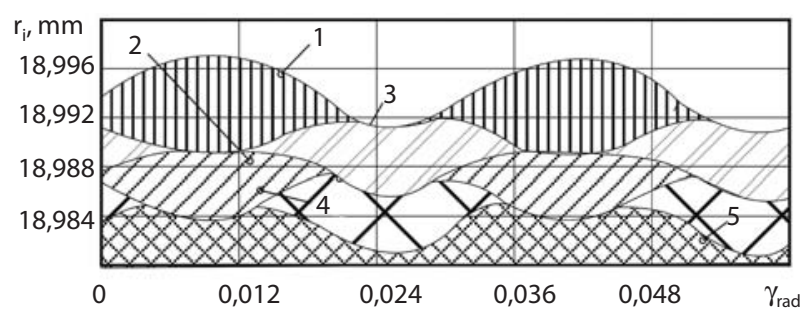

b)

Fig. 3. Forming of buckling on the processing surface: $a$ - for phase $0 ; b-$ for phase $\pi(1,2,3 . .-$ serial number of billet reverse side)

$S_{e n}(\omega)=\int_{-\infty}^{\infty} K_{R}(\gamma) \cdot \exp (-j \cdot \pi \cdot \omega) d \gamma$

The operator (26) is linear for assistive correlation functions, what allows to build component-by-component images of their spectra and to analyze the established reaction of the technological system (3) on separate fluctuation components.

The spectral parameters of forced oscillations of a grinding wheel center are depended on influence of random process of tool shape deviation during its interaction with a billet in the pro-cess of technological grinding and are determined, e.g., by the following relationship [20]:

$S_{e x}(\omega)=S_{e n}(\omega) \cdot\left|W_{T C}(j \omega)\right|^{2}$,

where $\left|W_{T C}(j \omega)\right|^{2}$ - squared module of transition function of dynamic parameters for a technological system; $S_{e n}(\omega)-$ spectral image of tool shape deviation, determined as Fourier transformation for correlation functions which are built on the base of the equation (26).

\section{Results}

The obtained spectra characterize energy distribution for forced oscillations of the technological system, which is induced be grinding wheel shape deviations. These relationships make the base for synthesis of forming filters for automatic systems.
The obtained results also display that decrease of buckling height can be practically achieved on a tool working surface due to periodical varying of self oscillations frequency in the system "billet - centers". In this case the waves formed on a wheel during oscillations period $T_{C}$ (Fig. 2a), will be deleted in processing with increased period $T_{\mathrm{c}}^{\prime}=T_{\mathrm{c}}+\Delta T_{\mathrm{c}}$. It is caused by the fact that billet strikes to top of the waves, not troughs, and destructs them gradually (Fig. $2 \boldsymbol{b}$ ).

As a result, the appearance similar to wave "self crosscutting" takes place and the height of waves on the processing surface decreases (Fig. 3).

The value $\Delta T_{c}$, which is required for calculation of oscillations period $T_{c}$, is determined using analysis of the condition for providing minimal dispersion of the shape $K_{R}\left(\gamma_{1}-\gamma_{2}\right)$ (27); this value should be located within a quarter of oscillations period, i.e. $0<T_{c}<T_{c} / 4$.

Maximal time interval between recurrent variations of the system self oscillations frequency depends on concrete processing conditions and is determined by critical allowable waves height on a wheel, which provides quality parameters of the processed components.

\section{Experimental testing of the results}

Experimental testing of the method was executedon the machine tool of ZE153 model, during grinding of 45 steel ring with HRC hardness 50-55 and dimensions $D \times d \times h=40 \times 25 \times 15 \mathrm{~mm}$. The wheel $1400 \times 40 \times 127$ 24A4OHCT26K was used, operation speed $V_{\kappa}=35 \mathrm{~m} / \mathrm{s}$, product speed $V_{u}=0.81 \mathrm{~m} / \mathrm{s}$, transversal feed $S_{u}=0.8 \mathrm{~mm} / \mathrm{min}$. Allowance for processing made $0.8 \mathrm{~mm}$ and average grinding 
time for one sample was $30 \mathrm{~s}$. The rigid centered plug $(e / d \approx 3)$ was used during processing. The period of self oscillations of the system "billet - centers" was measured via the standard technique [2] and made $T_{C}=0.83 \cdot 10^{-3} \mathrm{~s}$.

The first series of rings was processed via the conventional method, while the second series - using periodical variation of the period of self oscillations of the system "billet - centers". For this purpose the second billet (after tool correction) and all consequent even billets were subjected to grinding by wheels which were fixed on a plug with additional mass (disk $\Delta \mathrm{m}=0.65 \mathrm{~kg}$ ). At the same time the period of self oscillations increased by the value $0,25 \cdot T_{S}$ and made $1.05 \times 10^{-3} \mathrm{~s}$.

Amplitude of oscillations of $2 \mathrm{~A}_{I}$ centers during processing on a plug with constant mass in the end of resistance period ( $T=5 \mathrm{~min}$ ) exceeded $20 \mu \mathrm{m}$. Average buckling height of the wheel $W_{a v}$ made $17 \mu \mathrm{m}$. Grinding with variation of a plug mass showed that wheel buckling and oscillations remained practically permanent. The experiment was stopped after 15-20 min of wheel operation (after correction). Distinguished from the conventional method, no wobbling appearances were observed, periodical features of forced oscillations was not distinct. Quality of components processing was improved. Buckling height Wav decreased by 3 times, surface roughness $R_{a}$ lowered by 1.5 times. Surface burning were absent.

\section{Conclusion}

Preliminary simulation of the state of technological system during the period of wheel service life makes it possible to rise tool resistance, to decrease deviations of quality parameters, to increase operating reliability and efficiency via varying the dynamic system parameters (such as mass, damping, stiffness). It is mostly simple to vary self oscillations of the technological system in order to manage the process via stiffness or deduced mass of its mostly "weak" parts-billet and centers. It is possible to create the systems operating with preset periodical character of variation of oscillations and the systems with feedback.

\section{REFERENCES}

1. Malkin S., Guo C. Grinding Technology: Theory and Applications of Machining with Abrasives. New York: Industrial Press, 2008. 372 p.
2. Novoselov Yu. K. Dynamics of forming the surfaces during abrasive processing. Iz-datelstvo SevNTU. Sevastopol: 2012. 304 p.

3. Zhen Bing Hou, Ranga Komanduri. On the mechanics of the grinding process. Part I. Stochastic nature of the grinding process. International Journal of Machine Tools \& Manufacture. 2003. Vol. 43. pp. 1579-1593.

4. Korchak S. N. Productivity of grinding process for steel components. Moscow: Mashinostroenie. 1974. 280 p.

5. Lajmert P., Sikora V., Ostrowski D. A dynamic model of cylindrical plunge grinding process for chatter phenomena investigation. MATEC Web of Conferences. 2018. Vol. 148. pp. 09004. DOI: 10.1051/matecconf/20181480900.

6. Leonesio M., Parenti P., Cassinari A., Bianchi G., Monn M. A Time-Domain Surface Grinding Model for Dynamic Simulation. Procedia CIRP. 2012. Vol. 4. pp. 166-171. DOI: 10.1016/j. procir.2012.10.030.

7. Sidorov D., Sazonov S., Revenko D. Building a Dynamic Model of the Internal Cylindrical Grinding Process. Procedia Engineering. 2016. Vol. 150. pp. 400-405. DOI: 10.1016/j.proeng.2016.06.739.

8. Zhang N., Kirpitchenko I., Liu D. K. Dynamic model of the grinding process. Journal of Sound and Vibration. 2005. Vol. 280. pp. 425-432. DOI: 10.1016/j.jsv.2003.12.006.

9. Ahrens M., Damm J., Dagen M., Denkena B., Ortmaier T. Estimation of Dynamic Grinding Wheel Wear in Plunge Grinding. Procedia CIRP. 2017. Vol. 58. pp. 422-427. DOI: 10.1016/j. procir.2017.03.247

10. Garitaonandia I., Fernandes M. H., Albizuri J. Dynamic model of a centerless grinding machine based on an updated FE model. International Journal of Machine Tools \& Manufacture. 2008. Vol. 48. pp. 832-840. DOI: 10.1016/j.ijmachtools.2007.12.001.

11. Tawakolia T., Reinecke H., Vesali A. An Experimental Study on the Dynamic Behavior of Grinding Wheels in High Efficiency Deep Grinding. Procedia CIRP. 2012. Vol. 1. pp. 382-387. DOI: 10.1016/j.procir.2012.04.068.

12. Jung J., Kim P., Kim H., Seok J. Dynamic modeling and simulation of a nonlinear, non-autonomous grinding system considering spatially periodic waviness on workpiece surface. Simulation Modelling Practice and Theory. 2015. Vol. 57. pp. 88-99. DOI: 10.1016/j.simpat.2015.06.005.

13. Yu H., Wang J., Lu Y. Modeling and analysis of dynamic cutting points density of the grinding wheel with an abrasive phyllotactic pattern. International Journal of Advanced Manufacturing Technology. 2016. Vol. 86. pp. 1933-1943. DOI: 10.1007/s00170-0158262-0.

14. Guo J. Surface roughness prediction by combining static and dynamic features in cylindrical traverse grinding. International Journal of Advanced Manufacturing Technology. 2014. Vol. 75. pp. 1245-1252. DOI: 10.1007/s00170-014-6189-5.

15. Arriandiaga A., Portillo E., Sanchez J. A., Cabanes I., Pombo I. A new approach for dynamic modelling of energy consumption in the grinding process using recurrent neural networks. Neural Computing \& Applications. 2016. Vol. 27. pp. 1577-1592. DOI: $10.1007 / \mathrm{s} 00521-015-1957-1$.

16. Bratan S. M. Experimental researches of random dimensional parameters of a grinding wheel and building of analytical relationships for assessment of influence of its relief on input noise of grinding operation. Novye materialy $i$ tekhnologii $v$ metallurgii. Nauchnyi zhurnal ZGTU. 2000. Iss. 1. pp. 83-86. 